\title{
A new predictor is comparable to the updated nomogram in predicting the intermediate- and high-risk prostate cancer but outperforms
} nomogram in reducing the overtreatment for the
low-risk Pca

This article was published in the following Dove Press journal: Cancer Management and Research

\author{
Hui Wang ${ }^{1-3}$ \\ Sheng Tai ${ }^{1,2}$ \\ Li Zhang ${ }^{1,3}$ \\ Jun Zhou ${ }^{\text {I-3 }}$ \\ Chaozhao Liang ${ }^{1-3}$ \\ 'Department of Urology, The First \\ Affiliated Hospital of Anhui Medical \\ University, Hefei, People's Republic of \\ China; ${ }^{2}$ Anhui Province Key Laboratory \\ of Genitourinary Diseases, Anhui Medical \\ University, Hefei, People's Republic of \\ China; ${ }^{3}$ The Institute of Urology, Anhui \\ Medical University, Hefei, People's \\ Republic of China
}

Purposes: To develop a new predictor and update nomogram based on prostate imaging reporting and data system version 2 (PI-RADS V2) in predicting intermediate- and high-risk prostate cancer (IH-Pca) and reducing the overtreatment for low-risk Pca (L-Pca).

Methods: All men that underwent trans-rectal ultrasound-guided 12+X-core prostate biopsy between January 2015 and June 2018 were collected and analyzed. The significant risks (SRs) of Pca were selected by univariate and multivariate analysis. All SRs were divided into four groups ( 0 to 3 points) based on the probability of PI-RADS. Each patient can obtain a total score (TS). The updated nomogram was established by $\mathrm{R}$ package version 3.0. The area under the curve (AUC), net reclassification index (NRI), calibration curves and decision curves were used to evaluate the diagnostic performance.

Results: There were 1,078 patients, including 640 (59\%) men with normal or L-Pca (N-LPca) and $438(41 \%)$ men with IH-Pca. The scores of TS for IH-Pca and N-LPca were 16.13 \pm 3.11 and $10.52 \pm 3.32$, respectively $(P<0.01)$. The discriminative power of TS and nomogram was comparable in predicting IH-Pca (AUCs: 0.88 vs $0.87, P=0.89$ ), and both were greater than PSA and PIRADS (AUCs: 0.76 vs 0.80$)$. For NRI, NRI $\mathrm{TS}_{\text {vs nomogram }}$ was $1.31 \%(P=0.55)$, $\mathrm{NRI}_{\mathrm{TS}}$ vs PSA was $24.13 \%(P<0.001)$ and $\mathrm{NRI}_{\mathrm{TS}}$ vs PI-RADs was $13.19 \%(P<0.001)$. Compared with PSA, PI-RADS and nomogram, TS can reduce the number of unnecessary biopsies, up to $71 \%, 60 \%$ and $38 \%$, respectively.

Conclusion: The new predictor is comparable to the updated nomogram in predicting IH-Pca, and both are better than PSA and PI-RADS. In addition, the new predictor slightly outperforms nomogram in reducing the unnecessary biopsies for L-Pca and being convenient to use.

Keywords: age, PSA, PSA density, prostate cancer detection, PI-RADS, nomogram

\section{Introduction}

Many types of research suggested that men with clinically significant prostate cancer (CS-Pca) (Gleason score 3+4 or higher) need to be treated immediately, while those men with low-risk prostate cancer (L-Pca) can be selected to receive active surveillance (AS). ${ }^{1-4}$ At present, Pca diagnosis relies mainly on traditional detection measures, such as prostate-specific antigen (PSA), digital rectal examination (DRE) and magnetic resonance imaging (MRI). For men at risk of Pca, a systematic trans-rectal ultrasound
Department of Urology, The First

Affiliated Hospital of Anhui Medical

University, No. 218 Jixi Road,

Hefei 230022, Anhui,

People's Republic of China

Tel +8655I 62923440

Fax +86551 63633742

Email liang_chaozhao@ahmu.edu.cn 
(TRUS)-guided biopsy is a most common way to obtain the pathology for a definite diagnosis. Unfortunately, these traditional tests performed imperfectly in the diagnosis of Pca, especially in that of intermediate- and high-risk prostate cancer (IH-Pca), which resulted in a considerable number of unnecessary biopsies and overtreatment for men with normal or L-Pca (N-LPca). ${ }^{5}$

With the widespread use of traditional tests, we have noted that more and more men are undergoing unnecessary biopsies, and lots of men with L-Pca are founded. ${ }^{6,7}$ It is well known that L-Pca does not usually become a clinically relevant event in short periods of time when left untreated. Therefore, the diagnosis and subsequent treatment of L-Pca can also be called overdiagnosis and overtreatment. ${ }^{5}$ In brief, men with L-Pca do not need to be discovered in time, while those with IH-Pca, in contrary, should be taken seriously.

Previous studies have found that prostate imaging reporting and data system version 2 (PI-RADS v2) is an excellent measure to predict the probability of Pca and has better performance in identifying patients with CS-Pca. ${ }^{8}$ In addition, nomogram described in 1908 is an another way to predict the probability of Pca, with better performance than PSA and MRI in detecting indolent Pca and L-Pca. ${ }^{9,10}$ Nomogram is constituted by three parts, including the points of each risk, total points and risk probability. However, it is inconvenient to adopt nomogram in outpatient, and you might need to use a calculator or computer program to obtain the probability of risks. In view of this, our research is attempting to develop a new PI-RADS -based approach featuring higher predictive capability and more convenient use.

\section{Methods}

\section{Patients}

Patients with abnormal findings on PSA, DRE or MRI were definitely diagnosed by TRUS-guided $12+\mathrm{X}$-core prostate biopsy in our institute between January 2015 and June $2018(n=1,235)$. We collected the clinical data and retrospectively analyzed the relationship between the risks and IH-Pca. Previous studies reported by Presti were referred to for the steps and locations of biopsy. ${ }^{11}$ Pathological results were obtained by TRUS-guided biopsy, and part of the results was corrected by the pathology of radical prostatectomy (RP) for men undergoing RP at our center. In addition, we also collected the data from our database for men who underwent RP between
July 2018 and December 2018 to validate our new predictor $(n=96)$. The eighth edition of the TNM staging classification for prostate cancer was used to assign the clinical stage based on the multiparametric magnetic resonance imaging (MP-MRI), DRE and pathology. People with insufficient data were eventually excluded as follows $(n=157)$ : MRI $(n=91)$, free-PSA $(n=42)$, and the vague pathology $(n=24)$. A total of 1,078 evaluable patients were involved in establishing the new predictor and 96 patients were used to validate the performance of a new predictor.

This study was approved by Ethics Committees Regarding Human Research of the First Affiliated Hospital of Anhui Medical University (Approved ID: PJ-20170906). All experiments were in compliance with relevant guidelines and the Declaration of Helsinki. The data involved in this research was retrospectively collected from hospital electronic system and did not include the use of tissue samples and other samples, and the study did not include any personal information or privacy. For these reasons, it was not applicable to the informed consent of study participators, which was also exempted by the Ethics Committee.

\section{Risks collection and exclusion}

Pca risks were recommended by the Guidance on Diagnosis and Treatment of Urology of Chinese, including age, PSA, PSA density (PSAD), fPSA, \%fPSA, prostate volume (PV), DRE and MP-MRI. In addition, the valuable indicators that were previously reported by others were also collected, such as neutrophil, platelets, neutrophil to lymphocyte ratio (NLR) and body mass index (BMI). ${ }^{12,13}$

Univariate analysis was first used to filtrate indicators in order to obtain the independent risk factors of Pca. Next, multivariate stepwise regression analysis was employed to determine which factors are the independent risk factors for Pca in the model-building set. Finally, the satisfactory indicators were used to develop the models.

\section{Development of nomogram and new predictor}

$\mathrm{R}$ package version 3.0 (https://www.r-project.org) was adopted to build the nomogram relying on the satisfactory indicators. Then, total points and corresponding risk probability of Pca were calculated and utilized to predict the IH-Pca.

For new predictor, the values of all indicators were standardized by logarithmic transformation. Then, logistic regression was used to calculate the equation coefficients 
and OR, which were regarded as the weight coefficient of each indicator in the model. We used logistic regression to convert all indicators into the probability of predicting Pca. All indicators were divided into four groups: 1) unlikely to have Pca (PI-RADS 1 and 2 points); 2) equivocally (PIRADS 3 points); 3) likely (PI-RADS 4 points) and 4) highly likely (PI-RADS 5 points). The four groups scored differently depending on the possibility of Pca ( 0 to 3 , unlikely to highly likely). We calculated the probability of PI-RADS in the above four groups, and $95 \%$ CI of mean for other indicators that have the same probability interval with PIRADS was calculated and the upper bound of $95 \%$ CI was regarded as the boundary among groups.

The threshold of 0 -score group is the normal value of each indicator. In addition, for the age grouping, the normal group was aged 50 or below, which was recommended by Chinese Urological Guidelines. PSA grouping not only relied on the mean and $95 \%$ CI but also referenced the D'Amico risk group classification: low risk $(<10 \mathrm{ng} / \mathrm{mL})$, moderate risk $(10-20 \mathrm{ng} / \mathrm{mL})$ and high risk $(>20 \mathrm{ng} / \mathrm{mL}),{ }^{14}$ and we appropriately regulated the threshold of high risk to $26 \mathrm{ng} / \mathrm{mL}$ based on our data. Finally, according to the weight coefficient, the total score (TS) of new predictor for each patient is the sum of the product of each indicator's score with the weight coefficient, and TS was performed to predict the Pca or IH-Pca.

\section{Category and thresholds}

According to the D'Amico risk classification, the criteria of L-Pca are as follows: 1) PSA $<10 \mathrm{ng} / \mathrm{mL}$; 2) Gleason score $<7$ and 3) clinical stage $<$ T2b. Men with Pca who do not simultaneously meet the above conditions are regarded as having IH-PCa. The criteria of Prostate Cancer Research International Active Surveillance (PRIAS) are as follows: 1) PSA $\leq 10 \mathrm{ng} / \mathrm{mL}$; 2) Gleason score $<7$; 3) clinical stage $\leq \mathrm{T} 2 \mathrm{c}$; 4) 1 or 2 cores with Pca and 5) PSAD $\leq 0.2 \mathrm{ng} / \mathrm{mL}^{2}$. The criteria of significant prostate cancer are as follows: 1) tumor volume $>0.5 \mathrm{~mL}$; 2) extracapsular extension (ECE); 3) seminal vesicle invasions (SVI) and 4) Gleason score $\geq 7$.

All people were divided into two groups based on the pathology of biopsy and RP: IH-Pca and non-IH-Pca (normal or L-Pca). Besides, the cohort was also divided into the other two groups depending on the thresholds for predicting IH-Pca: negative test group and positive test group. The thresholds of a positive test are as follows: PSA $>10 \mathrm{ng} / \mathrm{mL}$, PI-RADS $>2$ points, PSAD $>0.15 \mathrm{ng} /$ $\mathrm{mLl}^{2}$, nomogram risk $>0.40$ and $\mathrm{TS}>13$.

\section{Statistical analysis}

Univariate analysis and multivariate stepwise regression analysis were used to filter indicators. To compare the performance of detecting Pca, we calculated the Youden's index, positive predictive value (PPV) and overall accuracy relying on the thresholds. PSA, PI-RADS, nomogram and TS as diagnostic methods were assessed in terms of the ability of predicting Pca and IH-Pca before biopsy by ROC curves and decision curve analysis (DCA) generated by Vickers. ${ }^{15}$ The area under the curve (AUC) was calculated and compared by $\mathrm{Z}$ test. The maximal point of the sum of sensitivity and specificity determined the optimal threshold. The net reclassification index (NRI) was calculated and tested by Z-test, which could reveal the performance in correctly reclassifying the patients. Calibration curves were utilized to show the extent of over- or underestimation of the predictive methods. Besides, we used the calibration with 1,000 bootstrap samples to decrease the overfit bias. Statistical significance was considered at $P<0.05$. The statistical analysis was performed with SPSS version 23.0 and package $\mathrm{R}$ version 3.0. (https://www.r-project.org).

\section{Results \\ Patient demographics}

In general, this study involved 1,078 patients, including 544 men with Pca (51\%), 534 men without Pca (49\%), 438 men with IH-Pca (41\%) and 640 men with N-LPca (59\%). The incidence of Pca and IH-Pca was rising with the age, PSA, PIRADS, nomogram and TS increasing, which were detailed in Table 1. Besides, we collected the data from our database for men that underwent RP to validate the new predictor and nomogram in predicting IH-Pca $(\mathrm{n}=96)$, including 19 patients with L-Pca and 77 patients with IH-Pca. The distribution of demographics and biopsy cores in different Gleason score, clinical stage and D'Amico risk is shown in Table 2. Men with IH-Pca can be definitely diagnosed with fewer biopsy cores and more positive cores. The biopsy cores for mean in all patients, normal patients and patients with Pca were 12.15, 12.38 and 11.92 , respectively, Table 2.

\section{Data collection and analysis}

The risks of Pca were collected, including age, PSA, PSAD, PV, fPSA, f/tPSA, DRE, PI-RADS, erythrocyte, neutrophil, NLR, lymphocyte, platelets and BMI. Then, $\mathrm{f} / \mathrm{t}$ PSA $(P=0.29)$, neutrophil $(P=0.89)$, lymphocyte $(P=0.48)$, NLR $(P=0.78)$ and BMI $(P=0.28)$ were excluded in univariate analysis, and PPSA $(P=0.09)$, DRE $(P=0.15)$, erythrocyte $(P=0.23)$ and platelets $(P=0.32)$ were excluded from the models in multivariate 
Table I The clinical characteristics, demographics and pathological findings of all men

\begin{tabular}{|c|c|c|c|c|c|c|}
\hline \multirow[t]{3}{*}{ Characteristic } & \multicolumn{4}{|c|}{ Training cohort $(\mathrm{N}=\mathrm{I}, 078)$} & \multicolumn{2}{|c|}{ Validation cohort $(\mathrm{N}=96)$} \\
\hline & \multirow[t]{2}{*}{ Total } & N-LPca & IH-Pca & \multirow[t]{2}{*}{$P$-value } & L-Pca & IH-Pca \\
\hline & & $n=640$ & $n=438$ & & $n=19$ & $n=77$ \\
\hline \multicolumn{7}{|l|}{ Mean(SD) } \\
\hline Total score & I $3.24(4.29)$ & $10.52(3.32)$ & $16.13(3.11)$ & $<0.01$ & $13.67(1.53)$ & $17.18(2.6)$ \\
\hline Nomogram risk & $0.41(0.32)$ & $0.2(0.2)$ & $0.63(0.28)$ & $<0.01$ & $0.44(0.19)$ & $0.72(0.24)$ \\
\hline Age, years & $68.90(8.28)$ & $67.06(8.26)$ & $70.91(7.84)$ & $<0.01$ & $72.33(8.5 \mathrm{I})$ & $71.9(7.23)$ \\
\hline tPSA level, $\mathrm{ng} / \mathrm{mL}$ & $30.95(30.90)$ & $17.24(\mid 7.68)$ & $45.88(35.04)$ & $<0.01$ & $7.32(2.68)$ & $53.91(36.23)$ \\
\hline $\mathrm{fPSA}, \mathrm{ng} / \mathrm{mL}$ & $6.59(11.49)$ & $3.36(6.57)$ & $10.1(14.33)$ & $<0.01$ & $0.89(0.17)$ & |3.8| (16.63) \\
\hline$\% \mathrm{fPSA}$ & $0.22(1.52)$ & $0.17(0.13)$ & $0.27(2.2)$ & 0.29 & $0.13(0.05)$ & $0.37(1.61)$ \\
\hline PSAD, $\mathrm{ng} / \mathrm{mL} / \mathrm{mL}$ & $0.74(0.90)$ & $0.39(0.5)$ & I.II (I.07) & $<0.01$ & $0.22(0.11)$ & 1.32 (I.37) \\
\hline $\mathrm{PV}, \mathrm{mL}$ & $53.87(36.22)$ & 57.79 (35.77) & $49.66(36.21)$ & 0.003 & $34.86(6.16)$ & 47.81 (30.73) \\
\hline Erythrocyte & $4.46(0.59)$ & $4.52(0.56)$ & $4.39(0.62)$ & 0.002 & $4.4 \mathrm{I}(0.28)$ & $4.36(0.66)$ \\
\hline Platelets & $196.48(65.02)$ & $200.5(68.59)$ & $192.03(60.6)$ & 0.073 & I55.67 (28.57) & I $88.87(66.01)$ \\
\hline Neutrophil & $4.06(3.4 I)$ & $4.07(1.78)$ & 4.04 (4.59) & 0.886 & $3.15(0.88)$ & $3.9(1.34)$ \\
\hline Lymphocyte & $1.73(2.3)$ & $1.67(0.73)$ & 1.79 (3.25) & 0.483 & $1.54(0.13)$ & $1.53(0.55)$ \\
\hline NLR & $2.88(2.58)$ & $2.86(2.18)$ & 2.91 (2.96) & 0.776 & $2.08(0.7 I)$ & $2.89(1.6)$ \\
\hline BMI & $23.35(3.37)$ & $23.48(3.07)$ & $23.22(3.66)$ & 0.283 & $22.43(2.95)$ & $22.89(3.23)$ \\
\hline \multicolumn{7}{|l|}{$\mathrm{N}(\%)$} \\
\hline \multicolumn{7}{|l|}{ DRE } \\
\hline Normal & $604(56)$ & $502(83)$ & $102(17)$ & $<0.01$ & II (58) & $8(42)$ \\
\hline Abnormal & $323(30)$ & $65(20)$ & $258(80)$ & $<0.01$ & $8(\mathrm{II})$ & $64(89)$ \\
\hline Unclear & $|5|(\mid 4)$ & $73(48)$ & $78(52)$ & $<0.01$ & 0 & $3(100)$ \\
\hline \multicolumn{7}{|l|}{ PI-RADS V2 } \\
\hline $\mathrm{I}-2$ & $224(2 I)$ & $211(94)$ & $13(6)$ & $<0.01$ & $2(40)$ & $3(60)$ \\
\hline 3 & $331(30)$ & $249(75)$ & $82(25)$ & $<0.01$ & $5(23)$ & $13(72)$ \\
\hline 4 & 306 (29) & I 46 (47) & $160(63)$ & $<0.01$ & $9(17)$ & $39(83)$ \\
\hline 5 & $217(20)$ & $34(16)$ & $183(84)$ & $<0.01$ & $3(12)$ & $22(88)$ \\
\hline
\end{tabular}

Abbreviations: N-Pca (Pca), non-Pca (prostate cancer); N-LPca, normal men and low-risk Pca; IH-Pca, intermediate- and high-risk Pca; $t$ (f)PSA, total (free) prostate-specific antigen; \%fPS, the ratio between tPSA and fPSA; PSAD, PSA density; PV, prostate volume; NLR, neutrophil to lymphocyte ratio; BMI, body mass index; DRE, digital rectal examination; PI-RADS V2, prostate imaging reporting and data system version 2 .

analysis. Finally, age, tPSA, PSAD, PV and PI-RADS $(P<0.05)$ were entered into the models by univariate analysis and further multivariate analysis.

\section{Nomogram and a new predictor (TS)}

Nomogram that consisted of age, tPSA, PSAD, PV and PIRADS was built, and the risk probabilities were calculated by $\mathrm{R}$ package, Figure $1 \mathrm{~B}$. The grouping and thresholds of a new predictor are shown in Table 3 and Figure 1A. Based on the equation coefficient and OR (Exp (B)), the ratio of the weight coefficient of age, PSA, PSAD, PV and PI-RADS was 1: 1: 1: 1: 3 , respectively. So, PI-RADS scored 3 times as many as other risks. The probability of PI-RADS including PI-RADS 1 and 2 points (normal), 3 points (equivocally), 4 points (likely) and 5 points (highly likely), respectively, was $\leq 15 \%, 15-40 \%$, $40-65 \%$ and $>65 \%$, and the mean and the $95 \%$ CI in the above four probability intervals were calculated (Table 3 ).
Each patient can get a TS ranging from 0 to 21 points. For IHPca, the screening threshold of TS was 14 points or higher (Figure 1A) and the threshold of nomogram was 0.40 or higher (Figure 1B).

\section{Comparison between TS and nomogram}

TS achieved the AUC of 0.88, which was comparable to nomogram (AUC: 0.87 ), $P=0.55$, and both of them were higher than the PSA and PI-RADS (AUCs: 0.79 and 0.80, respectively), $P<0.01$ (Tables 4 and 5, Figure 2A). There was no difference between TS and nomogram on the DCA, Figure $2 \mathrm{C}$. In addition, we also calculated the NRI, OR, PPV and overall accuracy to compare the performance of prediction between TS and nomogram, and the above results all suggested that the ability of TS in distinguishing the IH-Pca was similar to that of nomogram but outperformed the PSA and PI-RADS, Tables 4 and 5 . 
Table 2 Distribution of demographics and biopsy cores in different Gleason score, clinical stage and D'Amico risk

\begin{tabular}{|c|c|c|c|}
\hline \multirow[t]{2}{*}{ Characteristic } & \multirow{2}{*}{$\begin{array}{l}\text { Number of } \\
\text { patients (\%) }\end{array}$} & \multicolumn{2}{|l|}{ Mean (SD) } \\
\hline & & $\begin{array}{l}\text { Biopsy } \\
\text { cores }\end{array}$ & $\begin{array}{l}\text { Positive } \\
\text { cores }\end{array}$ \\
\hline Total & 1078 & $12.15(1.31)$ & $3.39(4.25)$ \\
\hline N-Pca & 534 (49) & $12.38(0.87)$ & - \\
\hline Pca & $544(5 \mathrm{I})$ & $11.92(1.60)$ & 6.59 (3.75) \\
\hline \multicolumn{4}{|l|}{ Gleason score } \\
\hline$\leq 6$ & $107(20)$ & $12.40(0.66)$ & $3.2(2.46)$ \\
\hline 7 & $160(29)$ & I2.0I (I.57) & 5.91 (3.47) \\
\hline$\geq 8$ & $277(5 \mathrm{I})$ & 11.69 (1.82) & $8.26(3.30)$ \\
\hline \multicolumn{4}{|l|}{ Clinical stage } \\
\hline$\leq \mathrm{T} 2 \mathrm{a}$ & $146(27)$ & 12.25 (1.25) & 2.18 (1.57) \\
\hline $\mathrm{T} 2 \mathrm{~b}$ & I55 (29) & $11.43(2.22)$ & 5.41 (1.65) \\
\hline$\geq T 2 c$ & $243(44)$ & $12.03(1.21)$ & $9.98(2.05)$ \\
\hline \multicolumn{4}{|l|}{ D'Amico risk } \\
\hline Low & $28(5)$ & $12.25(0.75)$ & $2.36(1.93)$ \\
\hline Intermediate & $113(21)$ & $12.50(0.94)$ & $4.10(2.8 \mathrm{I})$ \\
\hline High & $403(74)$ & 11.73 (1.75) & $7.58(3.55)$ \\
\hline
\end{tabular}

Abbreviation: N-Pca (Pca), non-Pca (prostate cancer).

In terms of reducing unnecessary biopsies and overtreatment for L-Pca, TS was better than nomogram, let alone for PSA and PI-RADS. For N-LPca men with negative test on TS, their positive tests on nomogram, PSA and PI-RADS accounted for $38 \%, 71 \%$ and $60 \%$, respectively (Figure 3A), which suggested that TS can prevent these men from biopsy. Besides, the distribution of men with N-LPca under the different screening methods was revealed by scatter diagram (Figure 3B) and the distribution of TS and nomogram risk in the different groups of D'Amico risks was shown by box plot (Figure 4A and B).

\section{Validation in cohort with RP}

In order to reduce the bias caused by biopsy pathology, we validated the performance of TS and nomogram in predicting Pca and significant Pca for people who underwent RP in our center between January 2015 and June 2018 ( $\mathrm{n}=332)$, including 221 men (66\%) with significant Pca and 111 men (34\%) with insignificant Pca. We found that TS was slightly better than nomogram in predicting the significant Pca (AUCs: 0.73 vs 0.70 ) and in predicting IH-Pca (AUCs: 0.88 vs 0.87 ), Figure $2 \mathrm{~B}$ and $\mathrm{D}$, and the overall accuracy of TS and nomogram were $85.1 \%$ and $83.8 \%$, respectively. Besides, we used a validation cohort ( $\mathrm{n}=96)$ to show the performance of a new predictor and nomogram in predicting IH-Pca. We also obtained excellent results, with AUC of 0.86 and 0.85 , respectively, and with an overall accuracy of $82.1 \%$ and $82.3 \%$ respectively.
In addition, we also evaluated the ability of TS and nomogram in selecting eligible men for AS by another criterion of PRIAS. Men who were normal or met the criteria of PRIAS were regarded eligible for AS. The results indicated that TS was comparable to nomogram with AUC of 0.88 and 0.87 , respectively (Figure 2E), and the overall accuracy of TS and nomogram was $78.9 \%$ and $78.4 \%$, respectively. Both were better than PSA (59\%) and PI-RADS (67\%).

\section{Calibration}

Calibration curves were used to illustrate the predicted consistency between actual risks and predictive risks, Figure 5A-D. From each calibration plot, we can know that TS and nomogram had the advantage of high goodness of fit. In other words, predictive risks of TS and nomogram were closely linked to the actual risks.

\section{Discussion}

At present, the consensus on Pca was early detection and treatment, ${ }^{6}$ which contributed to the more favorable prognosis. Therefore, widespread PSA screening was utilized to detect Pca in order to find Pca in time. Consequently, more and more men were undergoing unnecessary biopsies. ${ }^{5}$ Many studies have shown that L-Pca would not influence the lifetime and could be followed by AS rather than be treated immediately. ${ }^{3,4}$ If there is a predictive method that can identify L-Pca before biopsy, these men with L-Pca can avoid biopsy, and thus be relieved from the burden of economy and psychology. For men with IH-Pca, they should be diagnosed and treated as early as possible. Therefore, it is time to find out a way to identify L-Pca and IH-Pca. The new predictor has pretty decent ability in identifying IH-Pca and reducing overdiagnosis for normal and L-Pca men as well.

Nomogram was first described in 1908, and there were lots of studies about the performance of nomogram in predicting Pca. ${ }^{9,10,15-19}$ All these research had a common conclusion that nomogram outperforms any single predictor in forecasting Pca, such as PSA, PSAD, PI-RADS and so on. Besides, using nomogram to predict indolent Pca and CS-Pca was previously reported. ${ }^{10,15}$ From these research, we believe that nomogram has a bright prospect in the diagnosis and making treatment decision for Pca. However, there was no nomogram constituted by age, PSA, PSAD, PV and 


\section{A}

Total scores, TS:

Risk probability, \%:

For pca:

For IH-pca:

\begin{tabular}{|c|c|c|c|c|c|c|c|c|c|c|c|c|c|c|c|c|c|c|c|c|c|}
\hline 0 & 1 & 2 & 3 & 4 & 5 & 6 & 7 & 8 & 9 & 10 & 11 & 12 & 13 & 14 & 15 & 16 & 17 & 18 & 19 & 20 & 21 \\
\hline 0 & 0.3 & 0.5 & 0.7 & 1.0 & 1.6 & 2.5 & 4.1 & 6.4 & 10.0 & 15.3 & 22.7 & 32.3 & 43.7 & 55.7 & 67.1 & 76.8 & 84.3 & 89.7 & 93.4 & 95.8 & 97.4 \\
\hline & & & & & & & & & & & & & & & & & & & & & \\
\hline & & & & & & & & & & & & & & & & & & & & & \\
\hline
\end{tabular}

Negative test

Positive test

B

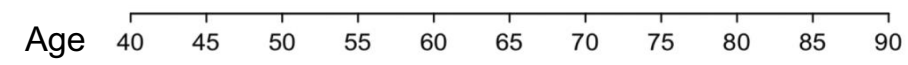

PSA

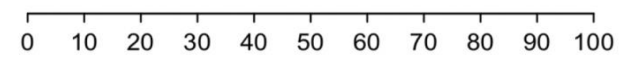

$\begin{array}{llllll}\text { PSAD } & 0 & 1 & 1 & 1 & 1 \\ & 0 & 3 & 6 & 9 & 12\end{array}$
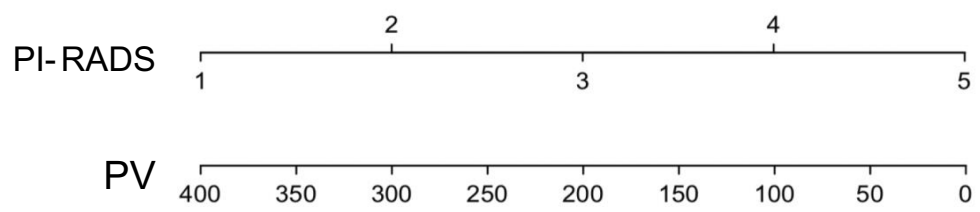

Total points

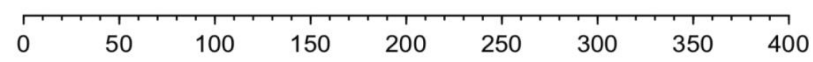

Risk

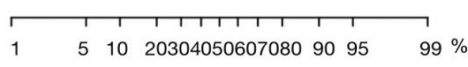

Figure I The thresholds for predicting Pca and IH-Pca and the updated nomogram. (A) The new predictor and screening thresholds. (B) The updated nomogram. Abbreviations: Pca, prostate cancer; IH-Pca, intermediate- and high-risk Pca; PSA, prostate-specific antigen; PSAD, PSA density; PV, prostate volume; PI-RADS, prostate imaging reporting and data system.

Table 3 The regression probability of PI-RADS in predicting Pca and the upper bound of $95 \% \mathrm{Cl}$ for mean under the regression probability of PI-RADS

\begin{tabular}{|c|c|c|c|c|}
\hline \multirow[t]{2}{*}{ Indicators } & 0 point & I point & 2 points & 3 points \\
\hline & Unlikely (normal) & Equivocally & Likely & Highly likely \\
\hline PI-RADS v2 & $\mathrm{I}-2$ & 3 & 4 & 5 \\
\hline Regression probability & $\leq 0.15$ & $0.15-0.40$ & $0.40-0.65$ & $>0.65$ \\
\hline Grouping boundary & & & & \\
\hline Main indicators & & & & \\
\hline PSA (ng/mL) & $0-4$ & $4-10$ & $10-26$ & $>26$ \\
\hline PSAD $\left(\mathrm{ng} / \mathrm{mL}^{2}\right)$ & $\leq 0.15$ & $0.16-0.20$ & $0.20-0.60$ & $>0.60$ \\
\hline PI-RADS* & $\mathrm{I}-2$ & 3 & 4 & 5 \\
\hline Auxiliary indicators & & & & \\
\hline Age (years) & $<50$ & $50-58$ & $59-70$ & $>70$ \\
\hline $\mathrm{PV}(\mathrm{mL})$ & $>454$ & $232-454$ & $42-232$ & $<42$ \\
\hline
\end{tabular}

Note: *This variable has 3 times the point of the other variables.

Abbreviations PSA, prostate-specific antigen; PSAD, PSA density; PV, prostate volume; PI-RADS v2, prostate imaging reporting and data system version.

PI-RADS in predicting IH-Pca for Chinese. Therefore, we used the above risks to establish a new nomogram, which demonstrated satisfying performance.
Apart from nomogram, there were other predicting tools for Pca detection, such as the European Randomized Study for Screening of Prostate Cancer Risk 
Table 4 Performance of PSA, PI-RADS, nomogram and TS in predicting the IH-Pca

\begin{tabular}{|c|c|c|c|c|}
\hline Evaluation indexes & PSA & PI-RADS & Nomogram & TS \\
\hline Youden's index & 0.32 & 0.45 & 0.56 & 0.57 \\
\hline Likelihood ratio & 170.97 & 285.36 & 388.13 & 398.75 \\
\hline PPV, \% & 59 & 64 & 70 & 71 \\
\hline OR $(95 \% \mathrm{Cl})$ & $7.82(5.50-\mid I .12)$ & $\begin{array}{l}14.42 \\
(9.89-21.02)\end{array}$ & $\begin{array}{l}16.19 \\
(11.76-22.30)\end{array}$ & $\begin{array}{l}20.11 \\
(14.03-28.82)\end{array}$ \\
\hline Overall accuracy,\% & 66 & 72 & 77 & 78 \\
\hline AUC $(95 \% \mathrm{Cl})$ for all cancer & $\begin{array}{l}0.75 \\
(0.72-0.78)\end{array}$ & $\begin{array}{l}0.80 \\
(0.78-0.83)\end{array}$ & $\begin{array}{l}0.87 \\
(0.85-0.89)\end{array}$ & $\begin{array}{l}0.87 \\
(0.85-0.89)\end{array}$ \\
\hline AUC $(95 \% \mathrm{Cl})$ for $\mathrm{GS}>6$ & $\begin{array}{l}0.77 \\
(0.74-0.80)\end{array}$ & $\begin{array}{l}0.81 \\
(0.79-0.84)\end{array}$ & $\begin{array}{l}0.87 \\
(0.85-0.89)\end{array}$ & $\begin{array}{l}0.88 \\
(0.85-0.89)\end{array}$ \\
\hline AUC $(95 \% \mathrm{Cl})$ for $\mathrm{Cs} \geq \mathrm{T} 2 \mathrm{~b}$ & $\begin{array}{l}0.79 \\
(0.76-0.82)\end{array}$ & $\begin{array}{l}0.80 \\
(0.77-0.83)\end{array}$ & $\begin{array}{l}0.87 \\
(0.84-0.89)\end{array}$ & $\begin{array}{l}0.87 \\
(0.85-0.89)\end{array}$ \\
\hline
\end{tabular}

Abbreviations: IH-Pca, intermediate- and high-risk Pca; PSA, prostate-specific antigen; PSAD, PSA density; PI-RADS, prostate imaging reporting and data system; TS, total scores; PPV, positive predictive value; AUC, area under the curve; GS, Gleason score; CS, clinical stage.

Table 5 The value of NRI, OR and AUC of predictive methods in predicting the IH-Pca

\begin{tabular}{|c|c|c|c|c|c|c|c|}
\hline \multirow{2}{*}{$\begin{array}{l}\text { Predictive } \\
\text { methods }\end{array}$} & \multicolumn{3}{|c|}{ Compared to TS } & \multicolumn{2}{|c|}{ Logistic regression } & \multicolumn{2}{|l|}{ For IH-Pca } \\
\hline & $\begin{array}{l}\text { NRI } \\
\%\end{array}$ & $Z$ value & $P$-value & $\begin{array}{l}\text { OR }(95 \% \\
\text { CI) }\end{array}$ & $P$-value & $\begin{array}{l}\text { AUC }(95 \% \\
\text { CI) }\end{array}$ & $\begin{array}{l}P \text {-value (compared to } \\
\text { TS) }\end{array}$ \\
\hline PSA & 24.13 & 4.58 & $<0.001$ & $\begin{array}{l}1.01 \\
(1.00-1.02)\end{array}$ & 0.024 & $\begin{array}{l}0.79 \\
(0.76-0.82)\end{array}$ & $<0.001$ \\
\hline PI-RADS & 13.19 & 6.97 & $<0.001$ & $\begin{array}{l}0.98 \\
(0.73-1.32)\end{array}$ & 0.91 & $\begin{array}{l}0.80 \\
(0.79-0.84)\end{array}$ & $<0.001$ \\
\hline Nomogram & 1.31 & 0.60 & 0.55 & $\begin{array}{l}15.68 \\
(3.43-71.73)\end{array}$ & $<0.001$ & $\begin{array}{l}0.87 \\
(0.86-0.90)\end{array}$ & 0.89 \\
\hline TS & - & - & - & $\begin{array}{l}16.87 \\
(4.25-67.00)\end{array}$ & $<0.001$ & $\begin{array}{l}0.88 \\
(0.87-0.90)\end{array}$ & - \\
\hline
\end{tabular}

Abbreviations: NRI, net reclassification index; TS, total score; IH-Pca, intermediate- and high-risk Pca; PSA, prostate-specific antigen; PSAD, PSA density; PI-RADS, prostate imaging reporting and data system; AUC, area under the curve.

Calculator, Korean Prostate Cancer Risk Calculator, Indonesian Prostate Cancer Risk Calculator and Prostate Cancer Prevention Trial Risk Calculator. These tools mainly consisted of age, PSA level, PV, family history, DRE and previous biopsies. ${ }^{20}$ Chinese Prostate Cancer Consortium Risk Calculator may be more suitable for Chinese, ${ }^{21}$ which includes risks of age, PSA, \%fPSA, PV and DRE. However, none of the above calculators used the latest PI-RADS studies to replace DRE, and the risk of PV was estimated by DRE based. Currently, MRI, in China, has been mainly used for identifying men with abnormal findings on DRE or elevated value of PSA, which is mainly related to its time and economic costs. PI-RADS $\mathrm{v} 2$ is based on the probability to predict Pca, including five stages ( 1 to 5 points). Many studies had suggested that PIRADS v2 has excellent ability in predicting Pca. ${ }^{22,23}$ So our research included PI-RADS in the updated nomogram and the new predictor, which highlighted the superiority of PI-RADS v2 in IH-Pca prediction and obtained decent values of AUC in forecasting IH-Pca (0.87 and 0.88).

Whether L-Pca men should be treated or not is controversial. As relevant researches go, the opinion that men with L-Pca should be followed by AS instead of being initially treated was accepted by more experts. ${ }^{3,4}$ Hayes carried out a comprehensive analysis of AS and initial treatment for L-Pca. ${ }^{4}$ The results suggested that AS was associated with the greatest quality-adjusted life expectancy (QALE), but individual preferences for receiving $\mathrm{AS}$ or treatment were highly related to QALE gains. Therefore, one of the aims of our research is to approximately assess whether suspected Pca men are with L-Pca. If so, we can consult with patients about their preference: undergoing a prostate biopsy to be definitely diagnosed or receiving AS at regular intervals. 


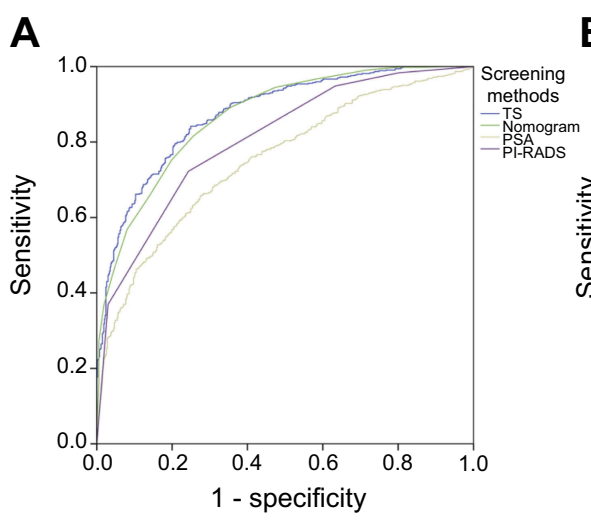

D

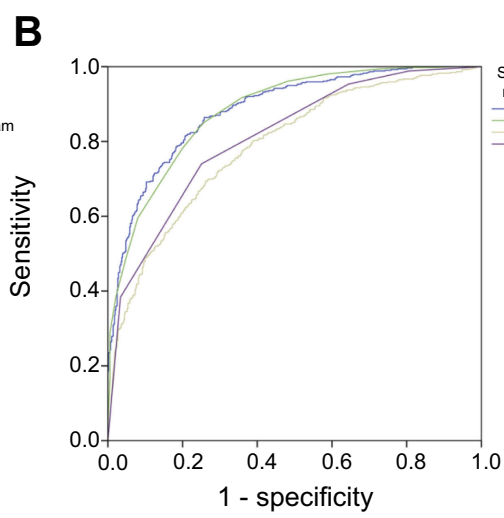

E

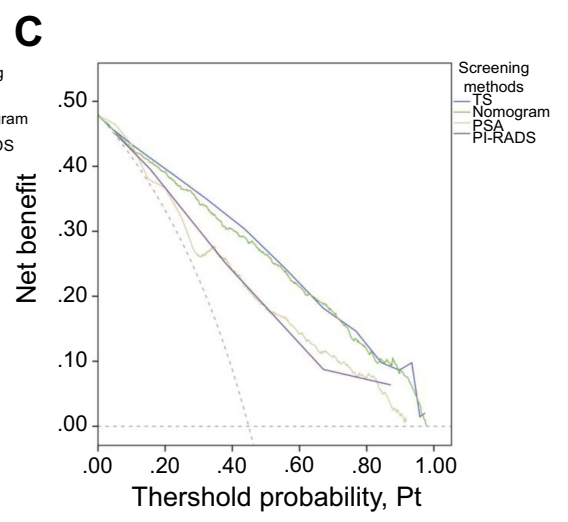

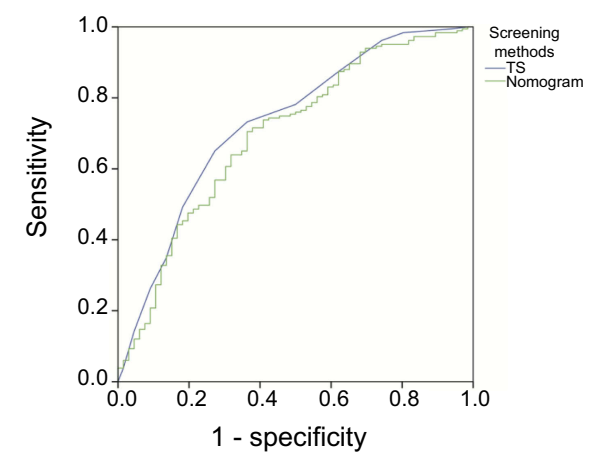

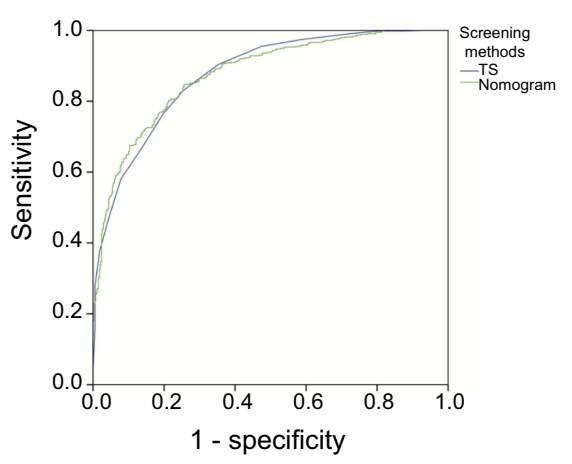

Figure 2 The predictive performance of different methods. (A) The area under the curve (AUC) is used to compare the predictive power in predicting IH-Pca. (B) The AUC is performed to show the predictive power in predicting $\mathrm{IH}-\mathrm{Pca}$ in population with radical prostatectomy. (C) The decision curve analysis is showing the predictive power in predicting IH-Pca. (D) The AUC is utilized to illustrate the performance of predicting significant Pca in cohort with radical prostatectomy. (E) Using AUC is to show the ability in forecasting the men following the criteria of PRIAS.

Abbreviations: Pca, prostate cancer; IH-Pca, intermediate- and high-risk Pca; PRIAS, Prostate Cancer Research International Active Surveillance.

If we can do that, not only the unnecessary biopsies will be greatly reduced but also patients' decision on treatment plan will be completely respected. In our research, both the new predictor and the updated nomogram have better ability to identify IH-Pca than PSA or PI-RADS alone.

Comparable to the updated nomogram in identifying IH-Pca, the new predictor has several advantages over the updated nomogram. First, the new predictor can reduce unnecessary biopsies for N-LPca men whose test of nomogram is positive, up to $38 \%$. Second, when using the nomogram to assess the probability of IH-Pca, we need to calculate a score for each indicator and add these score to get a TS.Based on the total scores, we obtain the probability of IH-Pca from the nomogram scale. The steps described above have to resort to computer program or scale-plate to calculate, because different values of indicators in the nomogram are corresponding to different scores. On the contrary, the new predictor is used according to the reference range to evaluate the probability of IH-Pca. Users only need to remember the value of boundary. In this sense, it is more convenient to use in the outpatient department.

Finally, our study also has several limitations. First, the study was a retrospective single-center study. Second, some people with PSA located on the gray area (PSA from 4 to $10 \mathrm{ng} / \mathrm{mL}$ ) refused to take further medical plan and preferred to choose follow-up observation, which made the number of these people decrease in the study. Third, although part of the pathological results was obtained from RP, most men with Pca were pathologically identified by TRUS biopsy, which may lead to a decrease in the number of IH-Pca so that the predictive accuracy of models would be underestimated. ${ }^{24,25}$

\section{Conclusion}

The new predictor is comparable to the updated nomogram in predicting the IH-Pca, and both are better than PSA and PI-RADS. In addition, the new predictor slightly outperforms nomogram in terms of reducing 
A

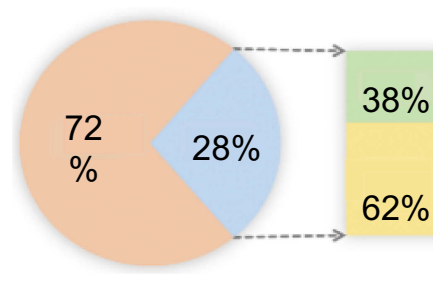

Nomogram (+)

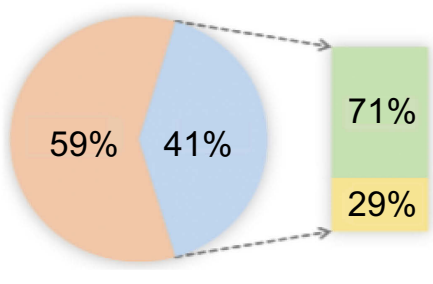

PSA (+)

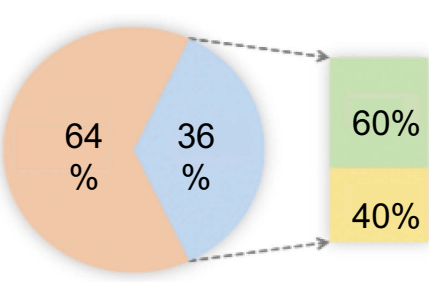

PI-RADS (+)

B

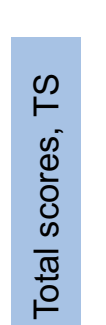

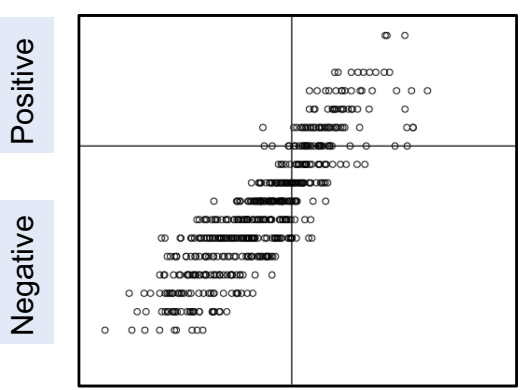

Negative Positive
N-LPca $\square$ TS test (-)

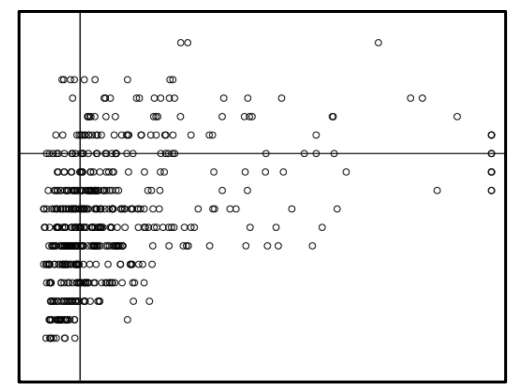

Negative

Positive

PSA test

TS test $(+)$

\section{Nomogram test}

Figure 3 The distribution of men with N-LPca and with IH-Pca in the different screening methods. (A) The distribution of TS for men with N-LPca or with IH-Pca in
positive tests of nomogram, PSA and PI-RADS. (B) The distribution of TS groups in different screening methods for men with N-LPca.
Abbreviations: N-LPca, normal men or low-risk prostate cancer; IH-Pca, intermediate- and high-risk Pca;TS, total score; PSA, prostate-specific antigen; PI-RADS, prostate imaging reporting and data system.

A

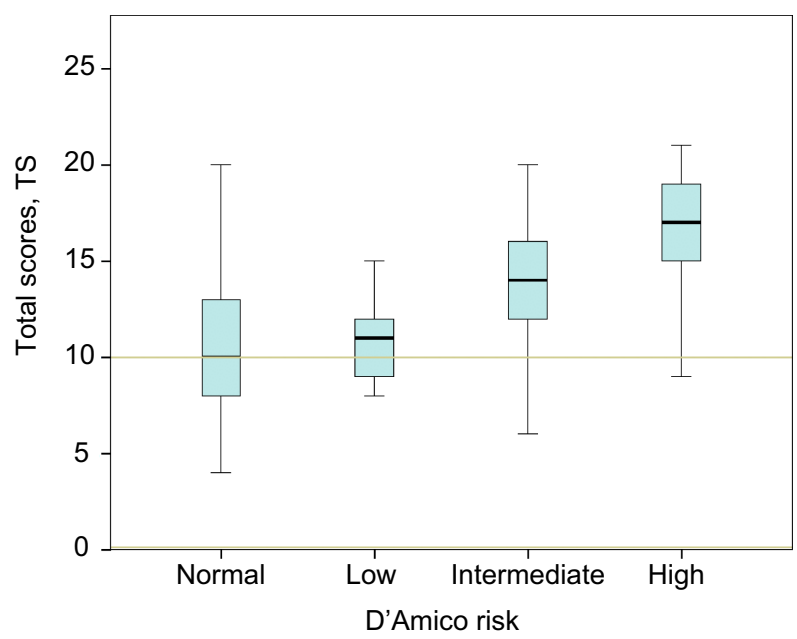

B

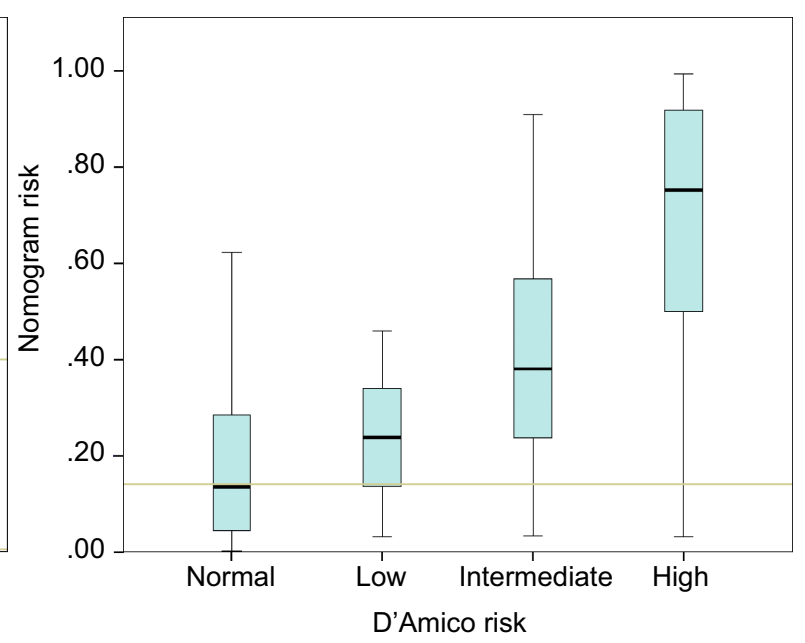

Figure 4 The value and mean of total scores and nomogram. (A) The value and mean of total scores in D'Amico risks. (B) The value and mean of nomogram in D'Amico risks.

unnecessary biopsies and being convenient to use. Therefore, this predictor is a promising approach to assisting doctors or patients in making a decision: receiving AS or having a biopsy immediately for men at risk of Pca.

\section{Acknowledgments}

This study was funded by the National Natural Science Foundation of China (No. 81630019, 81870519), Scientific Research Foundation of the Institute for Translational Medicine of Anhui Province (2017ZHYX02). 
A

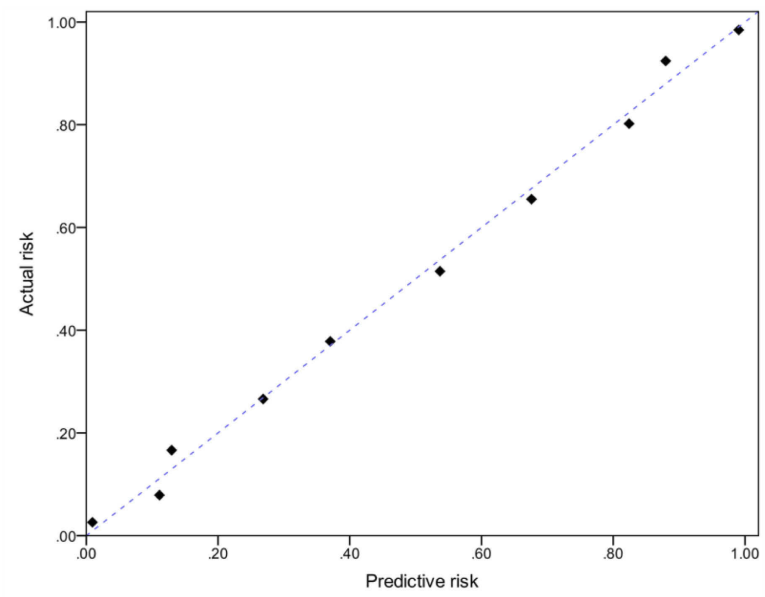

C

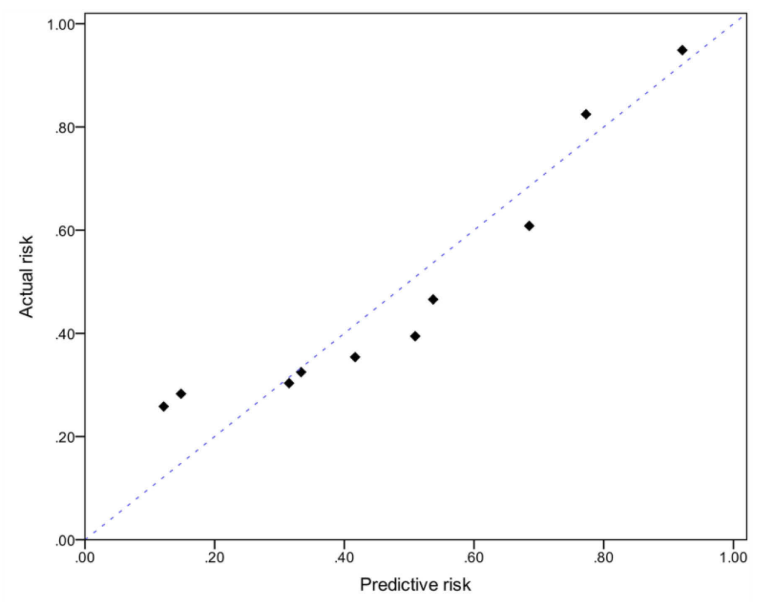

B

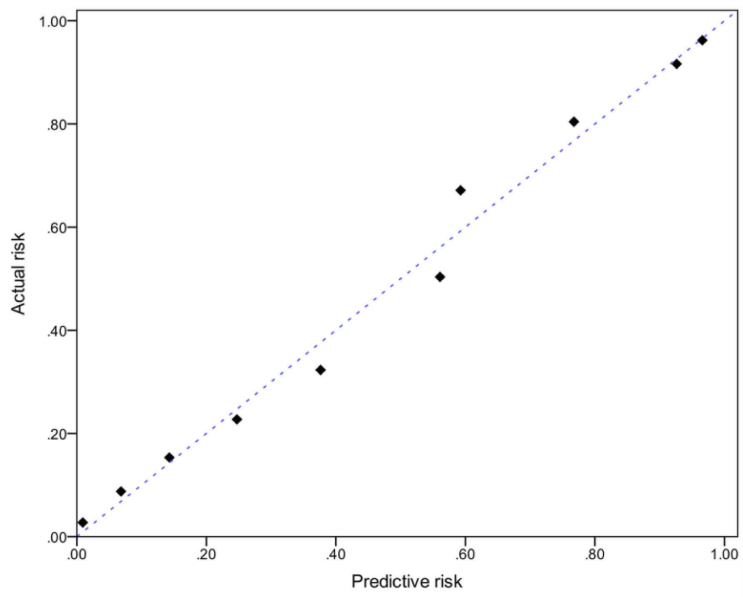

D

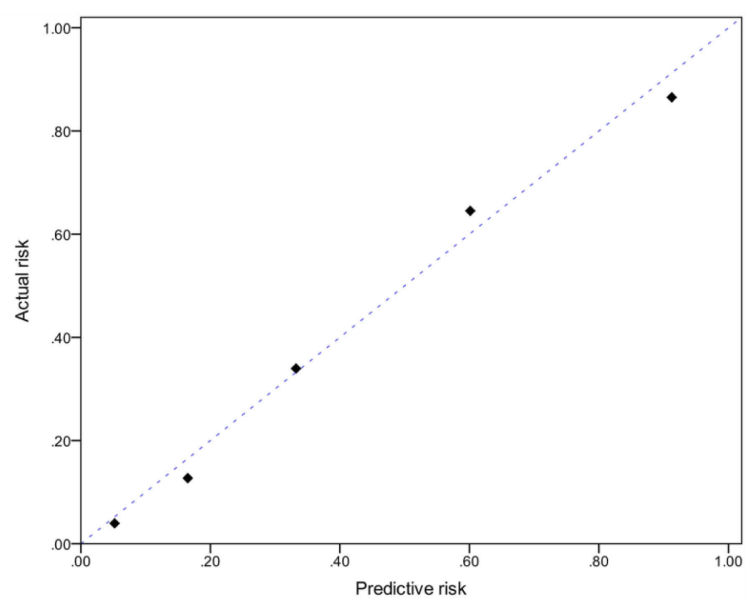

Figure 5 Calibration curves for the prediction models. (A) Total scores. (B) Nomogram. (C) PSA and (D) PI-RADS.

Abbreviations: PSA, prostate-specific antigen; PI-RADS, prostate imaging reporting and data system.

\section{Disclosure}

The authors report no conflicts of interest in this work.

\section{References}

1. Huynh MA, Chen MH, Wu J, Braccioforte MH, Moran BJ, D'Amico AV. Gleason score $3+5$ or $5+3$ versus $4+4$ prostate cancer: the risk of death. Eur Urol. 2016;69(6):976-979. doi:10.1016/j.eururo.2015.08.054

2. Hamdy FC, Donovan JL, Lane JA, et al. 10-year outcomes after monitoring, surgery, or radiotherapy for localized prostate cancer. N Engl J Med. 2016;375(15):1415. doi:10.1056/NEJMoa1606220

3. Dall MA, Bangma C, Carroll PR, et al. Active surveillance for prostate cancer: a systematic review of the literature. Eur Urol. 2012;62 (6):976-983. doi:10.1016/j.eururo.2012.05.072

4. Hayes JH, Ollendorf DA, Pearson SD, et al. Active surveillance compared with initial treatment for men with low-risk prostate cancer: a decision analysis. Eur Urol. 2011;59(5):882-883. doi:10.1016/j.eururo.2011.02.015

5. Schra FH, Hugosson J, Roobol MJ, et al. Screening and prostate cancer mortality: results of the European Randomised Study of Screening for Prostate Cancer (ERSPC) at 13 years of follow-up. Lancet. 2014;384 (9959):2027-2035. doi:10.1016/S0140-6736(14)60525-0
6. Chen W, Zheng R, Zeng H, Zhang S, He J. Annual report on status of cancer in China, 2011. Chin J Cancer Res. 2015;27(1):2-12. doi:10.3978/j.issn.1000-9604.2015.01.06

7. Nam RK, Saskin R, Lee Y, et al. Increasing hospital admission rates for urological complications after transrectal ultrasound guided prostate biopsy. J Urol. 2013;189(1):S12-S8. doi:10.1016/j.juro.2012.11.015

8. Park SY, Jung DC, Taik YT, et al. Prostate cancer: PI-RADS version 2 helps preoperatively predict clinically significant cancers. Radiology. 2016;280(1):106-116. doi:10.1148/radiol.16151133

9. Karakiewicz PI, Benayoun S, Kattan MW, et al. Development and validation of a nomogram predicting the outcome of prostate biopsy based on patient age, digital rectal examination and serum prostate specific antigen. J Urol. 2005;173(6):1930-1934. doi:10.1097/01. ju.0000158039.94467.5d

10. Roobol MJ, Kattan MW. Prediction of indolent prostate cancer: validation and updating of a prognostic nomogram. $J$ Urol. 2007;177(1):107-112. doi:10.1016/j.juro.2006.08.068

11. Presti JCP, Chang JJ, Bhargava V, et al. The optimal systematic prostate biopsy scheme should include 8 rather than 6 biopsies: results of a prospective clinical trial. J Urol. 2000;163(1):163-167.

12. Ferro M, Musi G, Serino A, et al. Neutrophil, platelets, and eosinophil to lymphocyte ratios predict gleason score upgrading in low-risk prostate cancer patients. Urol Int. 2019;102(1):43-50. doi:10.1159/000494259 
13. Cobelli O, Terracciano D, Tagliabue E, et al. Body mass index was associated with upstaging and upgrading in patients with low-risk prostate cancer who met the inclusion criteria for active surveillance. Urol Oncol. 2015;33(5):201.e1-201.e8. doi:10.1016/j.urolonc.2015.02.004

14. Boorjian SA, Karnes RJ, Rangel LJ, Bergstralh EJ, Blute ML. Mayo Clinic validation of the $\mathrm{D}$ ‘amico risk group classification for predicting survival following radical prostatectomy. $J$ Urol. 2008;179:1354-1361. doi:10.1016/j.juro.2007.11.061

15. Vickers AJ, Cronin AM, Elkin EB, Gonen M. Extensions to decision curve analysis, a novel method for evaluating diagnostic tests, prediction models and molecular markers. BMC Med Inform Decis Mak. 2008;8(1):53. doi:10.1186/1472-6947-8-53

16. Grivas N, Wit E, Tillier C, et al. Validation and head-to-head comparison of three nomograms predicting probability of lymph node invasion of prostate cancer in patients undergoing extended and/or sentinel lymph node dissection. Eur J Nucl Med Mol Imaging. 2017;44(13):2213-2226. doi:10.1007/s00259-017-3788-Z

17. Niu XK, He WF, Zhang Y, et al. Developing a new PI-RADS v2-based nomogram for forecasting high-grade prostate cancer. Clin Radiol. 2017;72(6):S0009926016305086. doi:10.1016/j.crad.2016.12.005

18. Greene DJ, Elshafei A, Nyame YA, et al. External validation of a PCA-3-based nomogram for predicting prostate cancer and highgrade cancer on initial prostate biopsy. Prostate. 2016;76 (11):1019-1023. doi:10.1002/pros.23197

19. Iremashvili V, Soloway MS, Pelaez L, Rosenberg DL, Manoharan M. Comparative validation of nomograms predicting clinically insignificant prostate cancer. Urology. 2013;81(6):1202-1208. doi:10.1016/j. urology.2013.01.062
20. Foley RW, Maweni RM, Gorman L, et al. The ERSPC risk calculators significantly outperform the PCPT 2.0 in the prediction of prostate cancer: a multi-institutional study. BJU Int. 2016;15 (3):706-713. doi:10.1111/bju.13437

21. Rui C, Xie L, Wei X, et al. Development and external multicenter validation of Chinese Prostate Cancer Consortium prostate cancer risk calculator for initial prostate biopsy. Urol Oncol. 2016;34 (9):416.e1-.e7. doi:10.1016/j.urolonc.2016.04.004

22. Seo JW, Shin SJ, Taik Y, et al. PI-RADS Version 2: detection of clinically significant cancer in patients with biopsy gleason score 6 prostate cancer. AJR Am J Roentgenol. 2017;209(1):W1-W9. doi:10.2214/AJR.16.16981

23. Washino S, Okochi T, Saito K, et al. Combination of prostate imaging reporting and data system (PI-RADS) score and prostatespecific antigen (PSA) density predicts biopsy outcome in prostate biopsy naive patients. BJU Int. 2016;119(2):225-233. doi:10.1111/ bju. 13465

24. Helfand BT, Loeb S, Kan D, Catalona WJ. Number of prostate cancer risk alleles may identify possibly 'insignificant' disease. BJU Int. 2010;106(11):1602-1606. doi:10.1111/j.1464-410X.2010.0 9440.x

25. Miyake H, Sakai I, Harada KI, Hara I, Eto H. Prediction of potentially insignificant prostate cancer in men undergoing radical prostatectomy for clinically organ-confined disease. Int J Urol. 2005;12(3):270-274. doi:10.1111/j.1442-2042.2005.0 1041.x
Cancer Management and Research

\section{Publish your work in this journal}

Cancer Management and Research is an international, peer-reviewed open access journal focusing on cancer research and the optimal use of preventative and integrated treatment interventions to achieve improved outcomes, enhanced survival and quality of life for the cancer patient.

\section{Dovepress}

The manuscript management system is completely online and includes a very quick and fair peer-review system, which is all easy to use. Visit http://www.dovepress.com/testimonials.php to read real quotes from published authors. 